\title{
Anomalous Medial Branch of Radial Artery: A Rare Variant
}

\author{
Surbhi Wadhwa*, Vandana Tomar
}

Department of Anatomy, University College of Medical Sciences \& GTB Hospital, Dilshad Garden, Delhi, India * Corresponding author: Department of Anatomy, Maulana Azad Medical College, New Delhi, India; e-mail: wadhwa. surbhi@gmail.com

Summary: Radial artery is an important consistent vessel of the upper limb. It is a useful vascular access site for coronary procedures and its reliable anatomy has resulted in an elevation of radial forearm flaps for reconstructive surgeries of head and neck. Technical failures, in both the procedures, are mainly due to anatomical variations, such as radial loops, ectopic radial arteries or tortuosity in the vessel. We present a rare and a unique anomalous medial branch of the radial artery spiraling around the flexor carpi radialis muscle in the forearm with a high rising superficial palmar branch of radial artery. Developmentally it probably is a remanent of the normal pattern of capillary vessel maintenance and regression. Such a case is of importance for reconstructive surgeons and coronary interventionists, especially in view of its unique medial and deep course.

Keywords: Radial artery; Variation; Loop/Anastomosis; Superficial palmar branch of radial artery; Flexor carpi radialis

\section{Introduction}

The main vessel of the upper limb, the brachial artery, divides at the level of the neck of the radius into two terminal branches - the radial and ulnar arteries. These arteries through further branching supply the forearm and hand along with the associated joints.

The widespread use of the forearm flap of the radial artery (RA) for reconstructive surgeries of head and neck as well as use of the RA as a route for transradial coronary intervention (TRI) has resulted in an interest in the variations of RAs. During reconstructive surgeries it is the aberrant anatomy which may affect the surgical outcome. Anomalies in the RA system although rare include hypoplasia, stenosis, tortuous configuration or presence of a radioulnar loop (3, 25). However the most frequently encountered anomaly is the high origin of RA or a superficial RA which accounts for $78 \%$ of all variations of RA (23).

We describe a rare anomalous medial branch of RA forming an unusual rectilinear anastomosis between the proximal RA and superficial palmar branch of RA (SPbr) coursing around the flexor carpii radialis muscle (FCR).

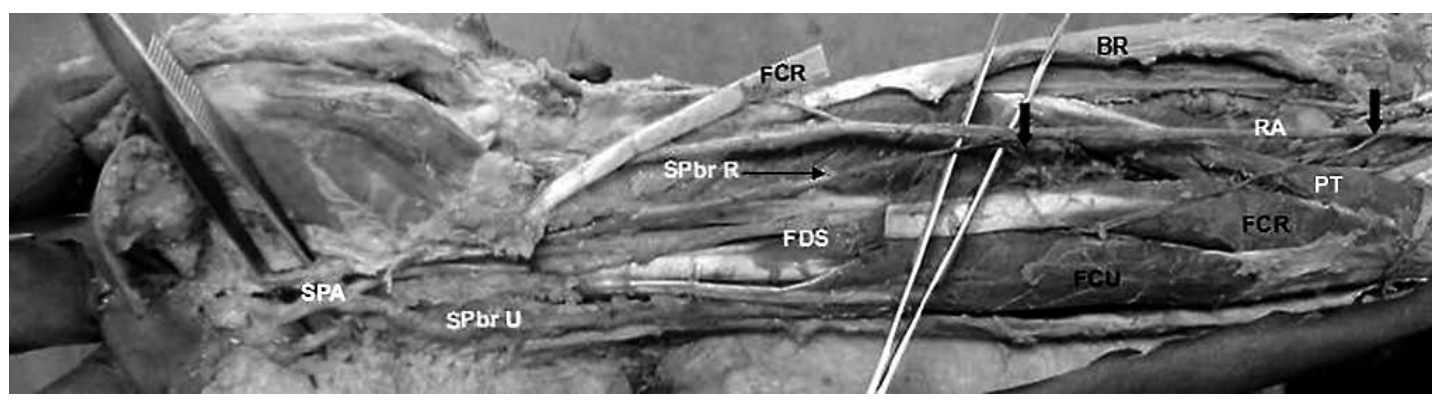

Fig. 1: Photograph of the dissection of the right forearm depicting the anomalous long slender branch (arrow), arising from the medial aspect of radialartery (RA), running anterior to the belly of flexor carpii radialis (FCR). Note the vessel courses posterior to the muscle, spirals around it and emerging at its lateral border to anastomose (arrow) to the high rising superficial palmar branch (SPbr), forming an arterial sling around the FCR. FCR - Flexor carpii radialis, BR - Brachioradialis, PT - Pronator teres, FCU - Flexor carpii ulnaris, FDS - Flexor digitorium superficialis, SPbrU - superficial palmar branch of ulnar artery, SPA - superficial palmar arch. 


\section{Case Report}

While dissecting the right forearm of a 72-year-old male cadaver for undergraduate teaching we encountered a unique variation of RA. The RA arose normally from the brachial artery at the level of neck of the radius. It descended into the forearm medial to the FCR and anterior to pronator teres under cover of brachioradialis. Just distal to the elbow it gave rise to a normal radial recurrent artery which ascended to anastomose with radial collateral branch of profunda brachii. Distally, while still in the cubital fossa, it gave an anomalous long slender branch, which ran medial to the RA, anterior to the belly of FCR. Near the musculotendinous junction of FCR, the vessel coursed posterior to the muscle, spiraling around it, emerging at its lateral border to anastomose to the $\mathrm{SPbr}$, forming an arterial sling around the FCR. The SPbr itself, originated at a higher level from the RA in the middle third of the forearm. This artery descended in the forearm along the RA to the wrist. While the RA ran on to the dorsum of the wrist, the SPbr coursed through the thenar muscles to complete the superficial palmar arch with the ulnar artery, which was otherwise normal in location and branching (Figures 1, 2, 3). The left limb did not show any anomalies.

\section{Discussion}

The above mentioned case presented with a unique variation, wherein an anomalous medial branch arose from the

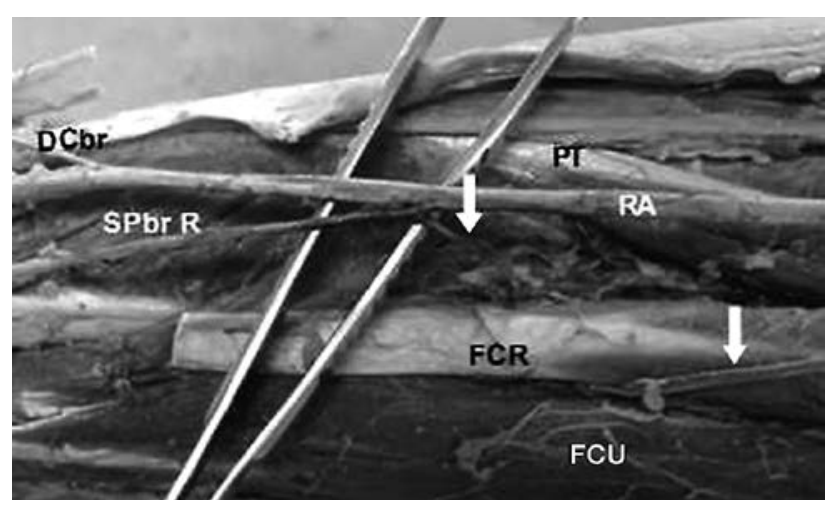

Fig. 2: Note the anastomoses of the anomalous medial branch to $\mathrm{SPbr}$ in the middle third of the forearm.

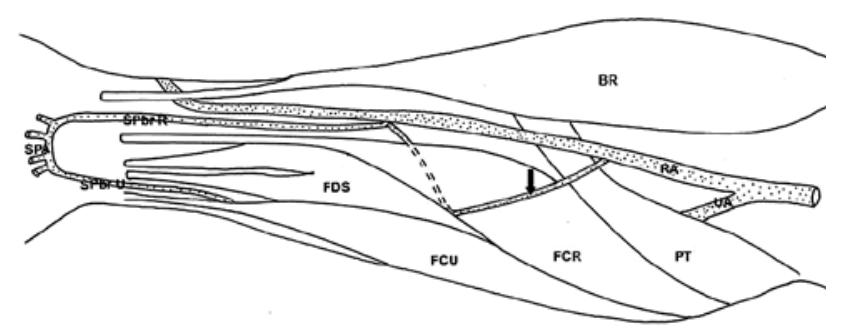

Fig. 3: Diagrammatic representation of the anomaly.
RA in the proximal part of the forearm, spiraled around the FCR and formed a rectilinear anastomosis with a high rising $\mathrm{SPbr}$, just distal to its origin from RA. The SPbr ran parallel to the RA in the distal third of the arm, entered the palm superficial to flexor retinaculm to form superficial palmar arch with normally arising superficial branch of an ulnar artery.

Anastomosis seen between vessels have been postulated to be atavistic remnants of embryological capillaries. They maybe between the superficial and deep set of vessels with an incidence of $1-6 \%$, mainly present around the elbow and are usually incidental findings $(14,17,18,19)$. Blood vessels of the upper limb are now postulated to arise from a capillary plexus in the limb bud which undergoes differentiation as it moves distally. While some of these capillaries are retained and undergo differentiation and enlargement, the others regress (20). These anastomosis have been classified on basis of their length (long/short), caliber (large/slender) and form (sling like/rectilinear) (17). Since they are present in the region of cubital fossa, another classification groups them on the basis of site of origin and number of recurrent radial arteries(121) or whether the anastomosis were present anterior or posterior to the bicipital tendon (14). In our case, the anastomosis is rectilinear in form, slings around FCR and is present in the forearm instead of the cubital fossa or arm.

Normally the RA, during its course through the forearm, gives rise to a radial recurrent artery just distal to the elbow in addition to muscular and cutaneous branches. This 'normal anatomy' of RA was seen in $92 \%$ of cases studied by Nasr (2012) in a gross cadaveric study. Commonly encountered anomalies of RA include variation in its course superficial or a deeply placed, variable origin, duplication or complete absence of the vessel or its septocutaneous perforator branches $(13,16,19)$. There are very few reports of anomalous branches arising from RA. These anomalous branches described in literature are laterally places and have a superficial course $(1,15,22)$. The only described medial branch was by Bhatt et al. (2009) which arose from the RA $1.5 \mathrm{cms}$ distal to its origin; however this vessel remained superficial to the FCR supplying the skin and fascia of the distal third of the forearm. Further description of the course of the vessel was not mentioned by the authors due to limited dissection during surgery. The aberrant branch in our case is a rare medial branch however in contrast to previousy reported case by Bhatt et al. (2009), this branch courses around the FCR and formed an anastomosis between RA and $\mathrm{SPbr}$ (2).

Honma et al., 2008, also found arterial rings but around the tendon of biceps brachii in the cubital fossa connecting the brachial artery to RA near its origin (6). While evaluating the impact of RA anomalies on transradial coronary intervention, loops were also observed by Lo et al. 2011. These loops were present between the RA and brachial artery/ulnar artery and mostly involved the proximal radial artery just below the brachial bifurcation. A recurrent radial artery (occasionally two) arose from the apex of the loop in all cases. 
The presence of these RA loops was associated with high procedural failure rate $(37.1 \%, \mathrm{p}=0.001)$.

Microvascular techniques help to effectively transfer the flap from its site of origin to donor repair site. However these techniques require accurate knowledge of the normal or variant anatomy of the forearm to avoid inadvertent injury or complications during and after the procedure. To raise the radial forearm flaps the surgeon needs to dissect carefully between the brachioradialis laterally and the FCR medially. It is here that a surgeon may encounter the anomaly mentioned here. The above mentioned anomaly also needs to be kept in mind while harvesting palmaris longus. The palmaris longus which lies medial to the FCR is also harvested for lip or mid-face reconstruction (7).

Inadvertent injury to the high rising SPbr as in this case when present should be borne in mind as ligation of it would compromise the superficial palmar arch especially in a handful of individuals where there is no communication between superficial and deep arches.

Nasr (2012), studied the origin, course and branching pattern of radial artery in 100 upper limbs wherein he found the SPbr to be variant in only 6 limbs. A high rising SPbr is a rare finding, with an incidence of $5-7 \%(4,16)$. The $\mathrm{SPbr}$ is a vessel which is consistently present in the forearm although they maybe variations associated with them. Flaps involving the SPbr of radial artery are used for forefinger reconstruction (8). A high arising SPbr running parallel to the RA may sometimes be mistaken for the parent vessel and cannulated during invasive blood pressure monitoring. Since radiocepahalic arteriovenous fistulas are end (of the vein) to side (of the artery), such anomalies are unlikely to impact surgical technique or efficacy.

The radial artery, as a virtue of its superficial location which is away from the major nerves of the upper limb, is regarded as a useful vascular access site for coronary procedures like transradial coronary angiography and transradial coronary intervention. It is also one of the the preferred vessel for coronary artery bypass grafting as it offers long term patency (26). During these procedures the artery is cannulated $1-2 \mathrm{~cm}$ proximal to the radial styloid process. Difficulties arise due to presence of bilateral brachioradial arteries anastomosing with a brachial artery through a sling like loop (5), or due to radioulnar loop $(0.9 \%)$ or due to presence of tortuosities and variations $(9,10)$.The reported technical failure with transradial interventions is $1-5 \%$, attributed to variant anatomy, arterial spasms and inablity to puncture (10). These anomalies are not contraindications for interventions; however they result in difficulty in introducing guidewires especially in unidentified variant anatomy. Transradial coronary interventions when attempted on such patients result in increased risk of dissections and perforations $(11,25)$. It has been observed that the loop like anomalies when present in the limb can be circumvented with appropriate instrumentation and manupilation on the table but lead to increased patient discomfort which may sometimes hinder further catheter advancement and in some cases alternate routes needed to be explored (10). Lo et al., 2011 observed higher procedural failure rates in patients with radial artery anomaly (10). Keeping these risks in mind, it is advisable for the operating surgeon to routinely screen the upper limb for a detailed vessel anatomy prior to intervention.

Clinical tests for vascular patency, such as Allens Test would probably be unaffected in most instances. The close proximity of the RA and SPbr at the wrist would not allow selective compression and hence not change the result. However a Doppler study may indicate the presence of two vessels if used, as is increasingly the norm.

\section{Conclusion}

A medial anomalous branch of radial artery is a rare variation. Further its re-anastomosis to the high rising superficial palmar branch has not been reported before. Awareness of such vessel is of utility to the reconstructive and peripheral vascular surgeon during harvesting the radial artery or raising a radial flap.

\section{References}

1. Acarturk T, Newton E. Aberrant branch of radial artery encountered during elevation of radial forearm flap. J Reconstr Microsurg 2004; 20: 611-4.

2. Bhatt V, Green J, Grew N. Dealingwith aberrant vessels in radial foream flaps- Report of a case and review of literature. J CranioMaxilloFac Surg 2009; 37: 87-90.

3. Byung-Su Y, Junghan Y, Ji-Yean K, et al. Anatomical consideration of the radial artery for transradial coronary procedures: arterial diameter, branching anomaly and vessel tortuosity. Inter J Cardiol 2005; 101: 421-7.

4. Gupta C, Ray B, D'Souza AS, Nair N, Pai SR, Manju M. A morphological study of variations in the branching pattern and termination of the radial artery. Singapore Med J 2012; 53: 208-1.

5. Hong T, Qiuhong D, Haipeng C. Brachioradial arteries with anastomotic arteries connecting to brachial arteries bilaterally. Hellinic J Cardiol 2010; 51: 358-61.

6. Honma S, Tokiyoshi A, Katsushi K, Koisumi M, Kodama K. Radial artery running beneath the biceps tendon and its interrelation between the radial recurrent arteries. Anat Sci Int 2008; 83: 232-8.

7. Jeng SF, Kuo YR, Wei FC, Su CY, Chien CY. Total lower lip reconstruction with a composite radial forearm-palmaris longus tendon flap: a clinical series. Plast Reconstr Surg 2004; 113(Pt 1): 19-23.

8. Lee TP, Liao CY, Wu IC, Yu CC, Chen SG. Free flap from the superficial palmar branch of the radial artery (SPBRA flap) for finger reconstruction. J Trauma 2009; 66(Pt 4): 1173-9.

9. Li Lang, Zeng ZY, Zhong JM, et al. Features and variations of a radial artery approach in southern Chinese populations and their clinical significance in percutaneous coronary intervention. Chin Med J 2013; 126(Pt 6): 1046-52.

10. Lo TS, Nolan J, Fountzopoulos E, et al. Radial artery anomaly and its influence on transradial coronary procedural outcome. Heart 2009; 95: 410-5.

11. Louvard Y, Lefevre T. Loops and transradial approach in coronary diagnosis and intervention. Catheter Cardiovasc Interv 2000; 51:250-2.

12. Ljubomudroff AP. Zur Morphologie der Arterienanastomosen in der Fossa cubiti. Z Anat Entwicklungs 1927; 84: 795-813.

13. Madaree A, McGibbon IC. Anatomic variation in the blood supply of the radial forearm flap. J Reconstr Microsurg 1993; 9: 277-9.

14. McCormack LJ, Cauldwell EW, Anson BJ. Brachial and antebrachial arterial patterns; a study of 750 extremities. Surg Gynecol Obstet 1953; 96: 43-54.

15. Morris LG, Rowe NM, Delacure MD. Superficial dorsal artery of the forearm. Case report and review of the literature. Ann Plast Surg 2005; 55(Pt 5): 538-54.

16. Nasr The radial artery and its variations:anatomical study and clinical implications. Folia Morphol 2012; 71: 252-62.

17. Quain R. Anatomy of the Arteries of the Human Body. London: Taylor and Walton, 1844.

18. Rodriguez-Baeza A, Nebot J, Ferreira B, et al. An anatomical study and ontogenic explanation of 23 cases with variations in the main pattern of the human brachio antebrachial arteries. J Anat 1995; 187: 473-9.

19. Rodríguez-Niedenführ M, Sanudo JR, Vazquez T, Nearn L, Logan B, Parkin I. Anastomosis at the level of the elbow joint connecting the deep, or normal,brachial artery with major arterial variations of the upper limb. J Anat 2000; 196: 115-9. 
20. Rodríguez-Niedenführ M, Burton GJ, Deu J, Sañudo JR. Development of the arterial pattern in the upper limb of staged human embryos: normal development and anatomic variations. J Anat 2001; 199: 407-17.

21. Rodríguez-Niedenführ M, Sanudo JR, Vazquez T, Nearn L, Logan B, Parkin I Variations of the arterial pattern in the upper limb revisited: a morphological and statistical study, with a review of the literature. J Anat 2001; 199: 547-66.

22. Sasaki K, Nozaki M, Aiba H, Isono N. A rare variant of the radial artery: clinical considerations in raising a radial forearm flap. Br J Plast Surg 2000; 53(Pt 5): $445-7$.

23. Uglietta JP, Kadir S . Arteriographic study of variant arterial anatomy of the upper extremities. Cardiovasc Interv Radiol 1989; 12: 145-8.

24. Weinand C, Akbari C, O'Donnell S. A High Bifurcation of the Dorsal Branch with Dominant Superficial Palmar Branch of the Radial Artery: A Case Report of an
Aberrant Radial Artery with Traumatic Aneurysm. J Hand Microsurg 2011; 3(Pt 2): $78-81$.

25. Yokoyama N, Takeshita S, Ochiai M, et al. Anatomic variations of the radial artery in patients undergoing transradial coronary intervention. Catheter Cardiovasc Interven 2000; 49: 357-62.

26. Yoo BS, Yoon J, Ko JY, et al. Anatomical considerations of the radial artery for transradial coronary procedures: arterial diameter, branching anomaly and vessel tortousity. Int J Cardiol 2005; 101: 421-27.

Received: 26/04/2016

Accepted: 22/08/2016 